1990-05-01

\title{
Model-based estimation of wind fields over the ocean from wind scatterometer measurements. I. Development of the wind field model
}

David G. Long

david_long@byu.edu

Jerry M. Mendel

Follow this and additional works at: https://scholarsarchive.byu.edu/facpub

Part of the Electrical and Computer Engineering Commons

\section{Original Publication Citation}

Long, D. G., and J. M. Mendel. "Model-Based Estimation of Wind Fields Over the Ocean from Wind Scatterometer Measurements. I. Development of the Wind Field Model." Geoscience and Remote Sensing, IEEE Transactions on 28.3 (199): 349-6

\section{BYU ScholarsArchive Citation}

Long, David G. and Mendel, Jerry M., "Model-based estimation of wind fields over the ocean from wind scatterometer measurements. I. Development of the wind field model" (1990). Faculty Publications. 732. https://scholarsarchive.byu.edu/facpub/732 accepted for inclusion in Faculty Publications by an authorized administrator of BYU ScholarsArchive. For more information, please contact ellen_amatangelo@byu.edu. 


\title{
Model-Based Estimation of Wind Fields Over the Ocean From Wind Scatterometer Measurements, Part I: Development of the Wind Field Model
}

\author{
DAVID G. LONG, MEMBER, IEEE, AND JERRY M. MENDEL, FEllow, IEEE
}

\begin{abstract}
In this first of two papers, we develop a parametric model for near-surface mesoscale wind fields suitable for use in a model-based approach (described in Part II [26]) to simultaneously estimate the wind field over a large region of the scatterometer measurement swath from measurements of the radar backscatter of the ocean's surface. In this paper we describe the assumptions made in developing the model and evaluate the accuracy of the resulting model. The model is based only on scatterometer data and is computationally tractable, consisting of a linear equation relating the near-surface wind field to a vector of model parameters which is estimated from the scatterometer measurements. We consider tradeoffs in the accuracy of the model and the number of unknown parameters. While we have developed our wind field model expressly for wind field estimation from scatterometer measurements, it is our hope that these results will prove helpful in other research areas.
\end{abstract}

\section{INTRODUCTION}

$\mathrm{L}$ AUNCHED IN 1978, the NASA-sponsored experimental satellite known as SEASAT demonstrated, among other things, that winds over the ocean could be measured from space using a wind scatterometer [7], [12], [25]. The scatterometer measures the wind-dependent radar backscatter of the ocean's surface from which the speed and direction of the wind over the ocean's surface can be inferred.

In this paper we introduce a parametric descriptive model for near-surface mesoscale wind fields over the ocean that is suitable for use in a new estimation-theorybased approach to estimating the wind vector field from spaceborne scatterometer measurements. Our approach, described in Part II [26], uses this model for the underlying wind field. The scatterometer measurements are used to estimate the parameters of the wind field model, and then the wind field estimate is computed using the estimated model parameters. This approach is fundamentally different from the traditional point-wise approach to wind field estimation and yields more accurate estimates of the

Manuscript received February 16, 1989; revised December 7, 1989. D. G. Long is with the Jet Propulsion Laboratory, California Institute of Technology, MS 300-319, 4800 Oak Grove Drive, Pasadena, CA 91109.

J. M. Mendel is with the Signal and Image Processing Institute, Department of Electrical Engineering-Systems, University of Southern California, Los Angeles, CA 90089.

IEEE Log Number 9034617. wind field. Our companion paper describes the modelbased estimation approach and provides a comparison of the model-based and traditional point-wise approaches to wind field estimation.

In this paper we first describe our requirements for the wind field model, and then present the assumptions used to derive our very simple descriptive wind field model which represents near-surface mesoscale wind fields. This is followed by the mathematical development of the wind field model. Various modeling options are then explored and, finally, the ability of the resulting model to describe "realistic" near-surface mesoscale wind fields is evaluated.

\section{Model-Based Wind Field Estimation}

For a model-based wind field estimation we need a mathematical model for describing and/or representing the wind field. This model must be capable of representing near-surface mesoscale wind fields to the desired accuracy. Since other data sources are not always available, we require that the model be based only on scatterometer data. To be useful for wind field estimation, the wind field model must be computationally tractable and lend itself to a model parameter estimation formulation [14], [18]. Note that the model does not necessarily have to be based on time-dependent atmospheric dynamics, since the model is used only for describing a snapshot of the near-surface wind field and not for propagating winds.

In this paper we present a particularly simple wind field model which is based on the geostrophic equation and simplistic assumptions regarding the divergence and curl of the horizontal wind field. While our final model does introduce some wind field smoothing, this smoothing can be controlled by the selection of the model order. We show that this model is adequate for use in wind field estimation from scatterometer measurements. As part of our continuing research we are investigating more sophisticated models.

The role of the wind field model in model-based wind field estimation is to provide a description of the wind field over the scatterometer measurement swath at a fixed instant of time and at a resolution of from 25 to $50 \mathrm{~km}$. 


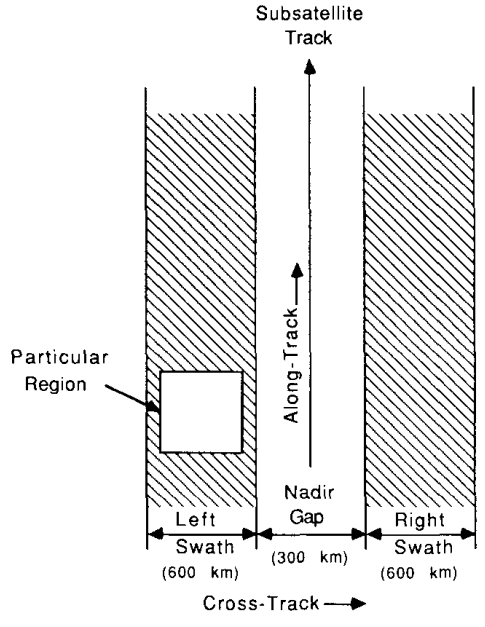

Fig. 1. An illustration of a typical (NSCAT) scatterometer measuremen swath. Observations of the radar backscatter are obtained on either side of the subsatellite track in the shaded region (see references [13] and [25]). A typical region for which the wind field model is applied is illustrated.

This corresponds to the scatterometer sampling resolution. We note that the scatterometer measurements of the radar backscatter are "averages" over the resolution "cells" of 25 to $50 \mathrm{~km}$ on a similarly spaced sample grid [6]. Thus the scatterometer measurements can only provide "smoothed" measurements of the wind field. On this basis we implicitly assume "mean field" approximation techniques in our wind field model development; our wind field model will be applicable for smoothed, sampled wind fields at the scatterometer resolution. To simplify matters we restrict our attention to limited-area regions with a maximum spatial extent of approximately $600 \mathrm{~km}$ (corresponding to the maximum scatterometer swath width for the planned NASA Scatterometer [NSCAT] [13]). For reference, Fig. 1 illustrates a typical region of interest within the NSCAT measurement swath.

Based on experience with the SEASAT scatterometer data, the Satellite Surface Stress $\left(S^{3}\right)$ working group [21] recommended wind estimate accuracy requirements for use in the next generation of scatterometers. Their requirements are summarized in Table I. Based on these requirements and the performance of the model-based wind estimation approach presented in Part II [26], only relatively mild requirements are needed on the accuracy of the wind field model. For example, an acceptable rootmean-square (rms) wind direction modeling error of $<6^{\circ}$ and an rms wind speed error of $<7.5 \%$ over the region are considered acceptable for use in scatterometer wind estimation. These mild requirements permit us to use a very simple parametric wind field model, one with only a small number of parameters. This simplifies the use of the model in the wind field estimation process.

\section{Wind Field Model Assumptions}

At the scatterometer resolution of 25 to $50 \mathrm{~km}$, the atmosphere can be considered to be essentially two-di-
TABLE I

Summary of The $S^{3}$ Wind Measurement ACCURACy Requirements for Future Spaceborne Wind Scatterometers

\begin{tabular}{cllc}
\hline \hline Wind Speed Range & Speed Accuracy & Direction Accuracy & Resolution \\
\hline$<3 \mathrm{~m} / \mathrm{s}$ & - & - & - \\
$3-6 \mathrm{~m} / \mathrm{s}$ & $\pm 2 \mathrm{~m} / \mathrm{s}$ & $\pm 20 \mathrm{deg}$ & $100 \mathrm{~km}$ \\
$6-100 \mathrm{~m} / \mathrm{s}$ & $\pm 2 \mathrm{~m} / \mathrm{s}$ or & $\pm 20 \mathrm{deg}$ & $\leq 50 \mathrm{~km}$ \\
& $10 \%$ of wind speed & & \\
\hline
\end{tabular}

mensional [23]. The scatterometer provides indirect measurements of the neutral stability near-surface wind at 19.5 $m$. Denote this near-surface horizontal wind field of interest by $\boldsymbol{U}=(u, v)^{T}$. We are interested in a mathematical model which provides a reasonably accurate description of $U$ over a (limited-area) region $\mathcal{L}$. The vorticity $\zeta$ and divergence $\delta$ of $\boldsymbol{U}$ are defined as

$$
\begin{aligned}
& \zeta=k \cdot \nabla \times U \\
& \delta=\nabla \cdot U .
\end{aligned}
$$

Following Lynch [17] and using the Helmholtz theorem, $U$ may be defined by a stream function $\psi$ and velocity potential $\chi$, according to

$$
\boldsymbol{U}=\boldsymbol{k} \times \nabla \psi+\nabla \chi
$$

where $k \times \nabla \psi$ is a nondivergent vector field and $\nabla \chi$ is a curl-free vector field [1].

Taking the divergence and curl, respectively, of (3), we obtain Poisson equations for $\psi$ and $\chi$ [17]:

$$
\begin{aligned}
& \nabla^{2} \psi=\zeta \\
& \nabla^{2} \chi=\delta .
\end{aligned}
$$

These equations appear in the classic problems of partitioning a given wind field into its rotational and nondivergent components and reconstructing a wind field from specified vorticity and divergence [1], [17]. For this latter problem, Lynch [17] argues that the reconstruction is not unique over a limited domain; an arbitrary harmonic function may be added to $\chi$, provided that $\psi$ is also altered, to produce the same wind field. From this he concludes that the boundary values of $\chi$ may be set arbitrarily. $\mathrm{He}$ shows that setting the boundary values of $\chi$ to zero minimizes the divergent component of the kinetic energy. Choosing $\chi=0$ on the boundary ensures a unique reconstruction of the wind field. Note, however, that this is not equivalent to assuming that the divergence is zero along the region boundary.

Based on this line of reasoning, our first modeling assumption is to assume that $\chi=0$ on the boundary of $\mathcal{L}$, which corresponds to assuming that the wind field has a minimum of divergent kinetic energy. Assuming that $\chi=$ 0 on the boundary, (4) and (5), the vorticity and divergence fields, and the boundary conditions for $\psi$ are sufficient for describing the wind vector field.

To obtain simple boundary conditions we make a second major modeling assumption by attributing $\psi$ to geostrophic motion. This second assumption is that the streamfunction $\psi$ is proportional to the geostrophic pres- 
sure field $p$; i.e.,

$$
\psi=\frac{1}{\rho_{s} f} p
$$

where $\rho_{s}$ is the density and $f$ is the Coriolis parameter [23]. This represents a departure from Lynch's [17] direct method for reconstructing a wind field from the normal velocity component along the boundary and the vorticity and divergence fields. Our approach allows further simplification of the model at a later step.

Note that in a strictly geostrophic formulation the wind field would be nondivergent and $\chi$ would be identically zero [23]. Mesoscale winds, however, may exhibit nonzero divergence; hence we adopt a more general formulation in which $\chi$ is not set to zero. Instead, $\chi$ is attributed to the ageostrophic component of the wind. This generalization allows us to apply the model to mesoscale wind fields that depart from strict geostrophy. Inclusion of the ageostrophic flow permits the model to span a wider space in describing the wind field, including fronts. Note that in applying the wind field model, $\psi$ and $\chi$ will be determined from the observed wind field.

By making our second modeling assumption we are able to specify the boundary values for (4) and (5) in terms of the geostrophic pressure field. This avoids the difficulties of using velocity boundary conditions that may yield an overdetermined system (see the discussion by Lynch [17]).

Our third modeling assumption is that, over our region of interest $\mathcal{L}, \rho_{s} f$ is essentially constant (i.e., an $f$-plane approximation [23]); we do this to simplify the mathematics. We can then normalize the pressure field by $\rho_{s} f$ so that $\psi=p$. Doing this, (3) can be written in component form as

$$
\begin{aligned}
& u=-\frac{\partial p}{\partial y}+\frac{\partial \chi}{\partial x} \\
& v=\frac{\partial p}{\partial x}+\frac{\partial \chi}{\partial y} .
\end{aligned}
$$

These two equations, along with (4) (in which $\psi=p$ ) and (5), form the basis of our wind field model.

To complete the wind field model, descriptions of the vorticity and divergence fields are needed. Our fourth and final modeling assumption is that the vorticity and divergence fields are relatively smooth at the scatterometer sampling resolution and vary relatively slowly over the region of interest $\mathcal{L}$; hence the vorticity and divergence fields can be modeled using only a small number of unknowns. This appears to be consistent with the limited data available [4], [5], [22]. We should point out that since the scatterometer inherently produces "smoothed" measurements of the wind, the corresponding vorticity and divergence fields can be expected to be "smooth."

Lacking a widely accepted model for the vorticity and divergence fields at the desired resolution, we have adopted a parameterization approach to modeling these fields. We have considered a number of different parameterizations of the vorticity and divergence fields [16] and have found that for our application (wind estimation from wind scatterometer measurements), bivariate polynomial approximations for these fields result in adequate accuracy; i.e.,

$$
\begin{aligned}
& \zeta(x, y) \triangleq \sum_{m=0}^{M_{C}} \sum_{\substack{n=0 \\
m+n \leq M_{C}}}^{M_{C}} c_{m, n} x^{m} y^{n} \\
& \delta(x, y) \triangleq \sum_{m=0}^{M_{D}} \sum_{\substack{n=0 \\
m+n \leq M_{D}}}^{M_{D}} d_{m, n} x^{m} y^{n}
\end{aligned}
$$

where $M_{C}$ and $M_{D}$ are the model orders and $c_{m, n}$ and $d_{m, n}$ are vorticity and divergence coefficients. Note that only the values of the vorticity and divergence polynomials at the scatterometer sample points are needed, and that the coefficients of these polynomials will be derived from the observed wind fields.

While the use of bivariate polynomials to model the vorticity and divergence fields is not physically based, this approach is simple and allows for tradeoffs in the model accuracy and number of unknowns. This approach also can directly provide the vorticity and divergence of the measured wind fields as well as permit us to control the geostrophy of the resulting wind fields. We have found, based on the results presented below, that $M_{C}=M_{D}=2$ is adequate for wind estimation from scatterometer measurements. However, more sophisticated models can be used. We are continuing to investigate alternative models for the vorticity and divergence fields.

\section{Model Development}

To further develop our simple wind field model for the purposes of wind field estimation from scatterometer measurements, we discretize (4), (5), and (7)-(10) on an $N \times N$ equally spaced grid with spacing $h$ over the region $\mathcal{L}$. For our purposes the value of $h$ is selected to correspond to the $25-50-\mathrm{km}$ sampling resolution of the wind scatterometer. The swath is segmented into abutting along-track regions (see Fig. 1). In the case of NSCAT, $N=24$ and $h=25 \mathrm{~km}$ will cover the entire left or right swath width [6], [13]. By further segmenting the swath into adjacent cross-track regions, $N$ may be chosen to be less than 24 . In this case, the $N h \times N h$ dimensions of the region $\mathscr{L}$ are reduced. We have found that choosing $N=$ 8 or 12 provides a good tradeoff between the number of unknowns in the model and the model's accuracy.

The discretization of (4), (5), and (7)-(10) is stable and will converge, assuming that the boundary conditions of the pressure field are bounded and have bounded higherorder derivatives [20].

We will show below that a simple linear equation can be used to relate the wind vector field at the sample points to the boundary conditions for $\psi$ (i.e., the geostrophic pressure field along the region boundary) and the parameters of the vorticity and divergence field models. 
Applying the first-order difference approximations [19],

$$
\begin{aligned}
& \left.\frac{\partial}{\partial x} a(x)\right|_{x=i h} \approx \frac{1}{h}\left[a\left(x_{i}\right)-a\left(x_{i-1}\right)\right] \\
& \left.\frac{\partial^{2}}{\partial x^{2}} a(x)\right|_{x=i h} \approx \frac{1}{h^{2}}\left[a\left(x_{i+1}\right)-2 a\left(x_{i}\right)+a\left(x_{i-1}\right)\right]
\end{aligned}
$$

to (4), (5), (7), and (8) and scaling by the discretization interval $h$, we obtain the following finite-difference equation (FDE) system:

$$
\begin{aligned}
u\left(x_{i}, y_{j}\right)= & -\left[p\left(x_{i}, y_{j}\right)-p\left(x_{i}, y_{j-1}\right)\right] \\
& +\left[\chi\left(x_{i}, y_{j}\right)-\chi\left(x_{i-1}, y_{j}\right)\right] \\
v\left(x_{i}, y_{j}\right)= & {\left[p\left(x_{i}, y_{j}\right)-p\left(x_{i-1}, y_{j}\right)\right] } \\
& +\left[\chi\left(x_{i}, y_{j}\right)-\chi\left(x_{i}, y_{j-1}\right)\right] \\
\zeta\left(x_{i}, y_{j}\right)= & p\left(x_{i+1}, y_{j}\right)+p\left(x_{i}, y_{j+1}\right)+p\left(x_{i-1}, y_{j}\right) \\
& +p\left(x_{i}, y_{j-1}\right)-4 p\left(x_{i}, y_{j}\right) \\
\delta\left(x_{i}, y_{j}\right)= & \chi\left(x_{i+1}, y_{j}\right)+\chi\left(x_{i}, y_{j+1}\right)+\chi\left(x_{i-1}, y_{j}\right) \\
& +\chi\left(x_{i}, y_{j-1}\right)-4 \chi\left(x_{i}, y_{j}\right)
\end{aligned}
$$

where $i=1, \cdots, N$ and $j=1, \cdots, N$, and where, for convenience, $\zeta\left(x_{i}, y_{i}\right)$ and $\delta\left(x_{i}, y_{i}\right)$ have been scaled by an additional factor of $h$. The boundary conditions for the $p$ field are the geostrophic pressure field $p\left(x_{0}, y_{j}\right)$ and $p\left(x_{N+1}, y_{j}\right)$ for $j=1, \cdots, N$ and $p\left(x_{i}, y_{0}\right)$ and $p\left(x_{i}\right.$, $y_{N+1}$ ) for $i=1, \cdots, N$ (refer to Fig. 2). The boundary conditions of the $\chi$ field are assumed to be zero.

For notational simplicity we write the discretized streamfunction (pressure field) $p\left(x_{i}, y_{j}\right)$ as $p_{i, j}$, where $x_{i}$ $=i h$ and $y_{j}=j h$. A similar notation will be used for the velocity, vorticity, divergence, and potential velocity fields.

Collecting the finite-difference equations for the streamfunction and potential velocity fields at each point of the square lattice covering $\mathcal{L},(15)$ and (16) can be written as two matrix equations; i.e.,

$$
\begin{aligned}
& Q P+P Q=\frac{1}{4} B+\frac{1}{4} C \\
& Q S+S Q=\frac{1}{4} D
\end{aligned}
$$

where $P, S, C$, and $D$ are $N \times N$ matrices with elements $p_{i, j}, \chi_{i, j}, \zeta_{i, j}$, and $\delta_{i, j}$, respectively; $Q$ is an $N \times N$ tridiagonal, symmetric, Toeplitz matrix with elements $q_{i, j}$, where,

$$
q_{i, J}=\left\{\begin{aligned}
\frac{1}{2}, & \text { if } i=j \\
-\frac{1}{4}, & \text { if }|i-j|=1 \\
0, & \text { otherwise }
\end{aligned}\right.
$$

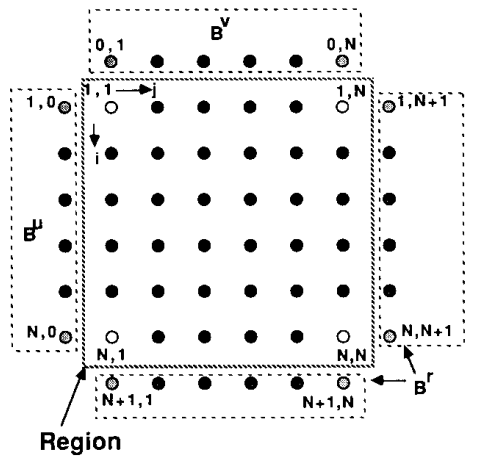

Fig. 2. Illustration of the model region boundary conditions.

and $B$ is a matrix containing only the $p$ field boundary values (the geostrophic pressure field $p$ along the boundary ). For later convenience we decompose $B$ into $3 N \times$ $N$ matrices:

$$
B=B^{v}+B^{u}+B^{r}
$$

where the elements of each matrix are

$$
\begin{aligned}
& b_{i, j}^{u}= \begin{cases}p_{i, 0} & \text { if } j=1, i=1, \cdots, N \\
0 & \text { otherwise }\end{cases} \\
& b_{i, j}^{v}= \begin{cases}p_{0, j} & \text { if } i=1, j=1, \cdots, N \\
0 & \text { otherwise }\end{cases} \\
& b_{i, j}^{r}= \begin{cases}p_{i, N+1} & \text { if } \leq i \leq N-1 \text { and } j=N \\
p_{N+1, j} & \text { if } i=N \text { and for } 1 \leq j \leq N-1 \\
p_{N, N+1} & p_{N+1, N} \\
& \text { if } i=N \text { and } j=N \\
0 & \text { otherwise. }\end{cases}
\end{aligned}
$$

Using an overbar to denote an $N^{2} \times 1$ vector of lexicographic-ordered (row order) elements of an $N \times N$ matrix, (17) and (18) can be reexpressed as

$$
\begin{aligned}
& K \bar{P}=\frac{1}{4} \bar{B}+\frac{1}{4} \bar{C} \\
& K \bar{S}=\frac{1}{4} \bar{D}
\end{aligned}
$$

where $I$ is the $N \times N$ identity matrix, and

$$
K \triangleq I \otimes Q+Q \otimes I
$$

is an $N^{2} \times N^{2}$ Toeplitz matrix, and $\otimes$ is the Kronecker product [3], [11]. It can be shown that $K$ is invertible and has a simple closed form [16].

Since $K$ is invertible, (24) and (25) have the unique solutions:

$$
\begin{aligned}
\bar{P} & =\frac{1}{4} K^{-1}[\bar{B}+\bar{C}] \\
& =\frac{1}{4} K^{-1}\left(\bar{B}^{u}+\bar{B}^{\prime \prime}+\bar{B}^{r}\right)+\frac{1}{4} K^{-1} \bar{C} \\
\bar{S} & =\frac{1}{4} K^{-1} \bar{D} .
\end{aligned}
$$


Starting with the first two equations of the FDE system, (13) and (14), reexpressed as

$$
\begin{aligned}
& u_{i, j}=-\left(\psi_{i, j}-\psi_{i, j-1}\right)+\left(\chi_{i, j}-\chi_{i-1, j}\right) \\
& v_{i, j}=\left(\psi_{i, j}-\psi_{i-1, j}\right)+\left(\chi_{i, j}-\chi_{i, j-1}\right)
\end{aligned}
$$

where $i=1, \cdots, N$ and $j=1, \cdots, N$, we can relate the $p$ and $\chi$ fields to the velocity field. To write (29) and (30) in matrix form, let $U$ and $V$ be $N \times N$ matrices with elements $u_{i, j}$ and $v_{i, j}$, respectively. These equations can then be written as

$$
\begin{aligned}
& U=\left[P\left(D_{1}-I\right)^{T}+B^{u}\right]+\left(I-D_{1}\right)^{S} \\
& V=\left[\left(I-D_{1}\right) P-B^{\prime \prime}\right]-S\left(D_{1}-I\right)^{T}
\end{aligned}
$$

where $D_{1}$ is an $N \times N$ matrix with elements $d_{i, j}^{1}$, which has a unity subdiagonal and is zero everywhere else; i.e.,

$$
d_{i, j}^{1}= \begin{cases}1, & \text { if } j=i-1 \\ 0, & \text { else. }\end{cases}
$$

Using lexicographic-ordered vectors, (31) and (32) can be written as

$$
\begin{aligned}
& \bar{U}=\left[G \bar{P}+\bar{B}^{u}\right]+H \bar{S} \\
& \bar{V}=\left[H \bar{P}-\bar{B}^{v}\right]-G \bar{S}
\end{aligned}
$$

where the $N^{2} \times N^{2}$ matrices $G$ and $H$ are defined as

$$
\begin{aligned}
& G \triangleq I \otimes\left[D_{1}-I\right] \\
& H \triangleq\left[I-D_{1}\right] \otimes I .
\end{aligned}
$$

Both $G$ and $H$ are full rank and invertible [16].

Note that $\bar{B}^{u}$ and $\bar{B}^{v}$ are $N^{2}$ element vectors with a maximum of $N$ nonzero elements, whereas $\bar{B}^{r}$ is an $N^{2}$ element vector with a maximum of $2 N-1$ nonzero elements. Consequently, there are a maximum of $4 N-1$ nonzero parameters in the $\bar{B}^{r}, \bar{B}^{u}$, and $\bar{B}^{v}$ vectors.

We note that the wind velocity is proportional to the partial derivatives (or, in this formulation, first-order differences) of the $p$ and $\chi$ fields. An arbitrary constant can be added to or subtracted from the $p$ and $\chi$ fields without affecting the results; hence a constant can be added to or subtracted from the boundary condition vectors. Since this additive constant is arbitrary and is unimportant, we can normalize the boundary condition vectors $\bar{B}^{u}, \bar{B}^{v}$, and $\bar{B}^{r}$ so that the first element of $\bar{B}^{u}, p_{0,1}$, is zero. This effectively eliminates one nonzero parameter, reducing the number of nonzero parameters in $\bar{B}^{u}, \bar{B}^{t}$, and $\bar{B}^{r}$ from $4 N$ - 1 to $4 N-2$.

Note also that the last element of $\overline{\boldsymbol{B}}^{r}$ is the sum of two boundary values $p_{N, N+1}$ and $p_{N+1, N}$; hence we do not need to separately identify these values, and so we need only identify the sum.

Substituting (27) and (28) into (34) and (35), we obtain

$$
\begin{aligned}
\bar{U}= & \frac{1}{4}\left[G K^{-1}\left(\bar{B}^{r}+\bar{B}^{v}+\bar{C}\right)+\left(G K^{-1}+4 I\right) \bar{B}^{u}\right] \\
& +\frac{1}{4} H K^{-1} \bar{D}
\end{aligned}
$$

$\bar{V}=\frac{1}{4}\left[H K^{-1}\left(\bar{B}^{r}+\bar{B}^{u}+\bar{C}\right)+\left(H K^{-1}-4 I\right) \bar{B}^{\prime \prime}\right]$

$$
-\frac{1}{4} G K^{-1} \bar{D} \text {. }
$$

To write (38) and (39) as a single equation, observe that they have the general form:

$$
\begin{aligned}
& \bar{U}={ }_{1} A\left(\bar{B}^{r}+\bar{C}\right)+{ }_{1} A \bar{B}^{v}+{ }_{2} A \bar{B}^{u}+{ }_{3} A \bar{D} \\
& \bar{V}={ }_{3} A\left(\bar{B}^{r}+\bar{C}\right)+{ }_{3} A \bar{B}^{u}+{ }_{4} A \bar{B}^{v}-{ }_{1} A \bar{D}
\end{aligned}
$$

where the ${ }_{j} A$ matrices are defined as

$$
\begin{aligned}
& { }_{1} A=\frac{1}{4} G K^{-1} \\
& { }_{2} A=\frac{1}{4}\left[G K^{-1}+4 I\right] \\
& { }_{3} A=\frac{1}{4} H K^{-1} \\
& { }_{4} A=\frac{1}{4}\left[H K^{-1}-4 I\right] .
\end{aligned}
$$

Let $\bar{X}$ be a $4 N-2$ element vector of the nonzero elements of $\bar{B}^{r}, \bar{B}^{u}$, and $\bar{B}^{v}$, where the $n$th element $\bar{x}_{n}$ of $\bar{X}$ is

$$
\bar{x}_{n}= \begin{cases}\bar{B}_{n+1}^{v} & 1 \leq n<N \\ \bar{B}_{(n-N) N+1}^{u} & N \leq n<2 N \\ \bar{B}_{(n-2 N+1) N}^{r} & 2 N \leq n<3 N \\ \bar{B}_{n-4 N+N^{2}+1}^{r} & 3 N \leq n \leq 4 N-2 .\end{cases}
$$

Define the $N^{2}$ element vector $\bar{W}$ as the concatenation of $\bar{U}$ and $\bar{V} ;$ i.e.,

$$
\bar{W}=\left[\begin{array}{c}
\bar{U} \\
\bar{V}
\end{array}\right] .
$$

Then the wind field model $((40)$ and (41)) can be expressed as the single equation:

$$
\bar{W}=F \bar{X}+R^{c} \bar{C}+R^{d} \bar{D}
$$

where $F$ is a $2 N^{2} \times(4 N-2)$ matrix and $R^{c}$ and $R^{d}$ are $2 N^{2} \times N^{2}$ matrices. $F, R^{c}$, and $R^{d}$ are composed of columns of the $A$ matrices in (42) through (45).

For convenience in defining $F$, we partition $F$ into four rectangular submatrices,

$$
F=\left[F_{1}\left|F_{2}\right| F_{3} \mid F_{4}\right]
$$

where the $F_{i}$ matrices are defined as

$$
\begin{aligned}
& F_{1}=\left[\begin{array}{llll}
\frac{{ }_{1} A_{2}}{{ }_{1} A_{3}} & \cdots & { }_{1} A_{N} \\
{ }_{4} A_{2} & { }_{4} A_{3} & \cdots & { }_{4} A_{N}
\end{array}\right] \\
& F_{2}=\left[\begin{array}{llll}
\frac{{ }_{2} A_{1}}{{ }_{2} A_{N+1}} & \cdots & { }_{2} A_{N^{2}-N+1} \\
{ }_{3} A_{1} & { }_{3} A_{N+1} & \cdots & { }_{3} A_{N^{2}-N+1}
\end{array}\right] \\
& F_{3}=\left[\begin{array}{llll}
\frac{{ }_{1} A_{N}}{{ }_{1} A_{2 N}} & \cdots & { }_{1} A_{N^{2}} \\
{ }_{3} A_{N} & { }_{3} A_{2 N} & \cdots & { }_{3} A_{N^{2}}
\end{array}\right] \\
& F_{4}=\left[\begin{array}{llll}
{ }_{1} A_{N^{2}-N+1} & { }_{1} A_{N^{2}-N+2} & \cdots & { }_{1} A_{N^{2}-1} \\
{ }_{3} A_{N^{2}-N+1} & { }_{3} A_{N^{2}-N+2} & \cdots & { }_{3} A_{N^{2}-1}
\end{array}\right]
\end{aligned}
$$


where ${ }_{j} A_{i}$ is the $i$ th column of the $j$ th $A$ matrix in (42) through (45). The matrices $F_{1}$ and $F_{4}$ are $2 N^{2} \times(N-$ 1 ), while $F_{2}$ and $F_{3}$ are $2 N^{2} \times N$. The matrix $R^{c}$ is defined as

$$
R^{c}=\left[\frac{{ }_{1} A}{{ }_{3} A}\right]
$$

whereas the matrix $R^{d}$ is defined as

$$
R^{d}=\left[\frac{{ }_{3} A}{-{ }_{1} A}\right] \text {. }
$$

Equation (48) provides a single matrix-vector equation relating the wind field velocity components contained in the $2 N^{2}$ element vector $\bar{W}$ to the $4 N-2$ element boundary condition vector $\bar{X}$ and the $N^{2}$ element vorticity and divergence field vectors $\bar{C}$ and $\bar{D}$, respectively.

Note that (48) can be expressed as

$$
\bar{W}=\bar{W}^{b}+\bar{W}^{c}+\bar{W}^{d}
$$

where

$$
\begin{aligned}
& \bar{W}^{b} \triangleq F \bar{X} \\
& \bar{W}^{c} \triangleq R^{c} \bar{C} \\
& \bar{W}^{d} \triangleq R^{d} \bar{D} .
\end{aligned}
$$

The wind field $\bar{W}$ can therefore be expressed as the sum of a field $\bar{W}^{b}$ which depends only on the boundary conditions in $\bar{X}$, a field $\bar{W}^{c}$ which depends only on the vorticity field in $\bar{C}$, and a field $\bar{W}^{d}$ which depends only on the divergence field in $\bar{D}$. Note that if we set $\bar{D}$ to be zero, we restrict the model to strictly geostrophic winds.

From our fourth modeling assumption we assume that the vorticity and divergence fields can be parameterized (or modeled) by a small number of unknown but deterministic parameters that are the coefficients of the bivariate polynomials in (9) and (10). Using this parameterization, the wind field model can then be formulated in terms of the boundary conditions on the $p$ field and the parameters of the vorticity and divergence field model. The number of parameters in the vorticity and divergence field models are $N_{C}=\left(M_{C}+1\right)\left(M_{C}+2\right) / 2$ and $N_{D}=$ $\left(M_{D}+1\right)\left(M_{D}+2\right) / 2$, respectively.

Using this polynomial parameterization for the vorticity and divergence fields, (48) can be written as

$$
\begin{aligned}
\bar{W}= & F \bar{X}+R^{c} \sum_{m=0}^{M_{C}} \sum_{\substack{n=0 \\
m+n \leq M_{C}}}^{M_{C}} c_{m, n} Q_{m, n} \\
& +R^{d} \sum_{m=0}^{M_{D}} \sum_{\substack{n=0 \\
m+n \leq M_{D}}}^{M_{D}} d_{m, n} Q_{m, n} \\
= & F \bar{X}+\sum_{m=0}^{M_{C}} \sum_{\substack{n=0 \\
m+n \leq M_{C}}}^{M_{C}} c_{m, n} R^{c} Q_{m, n} \\
& +\sum_{m=0}^{M_{D}} \sum_{\substack{n=0 \\
m+n \leq M_{D}}}^{M_{D}} d_{m, n} R^{d} Q_{m, n}
\end{aligned}
$$

where the $k$ th element ${ }_{k} q_{m, n}$ of the $N^{2}$ element vector $Q_{m . n}$ is

$$
{ }_{k} q_{m, n}=\lfloor k\rfloor^{m}+\lceil k\rceil^{n}
$$

in which $\lfloor k\rfloor \triangleq \operatorname{int}[(k-1) / N]+1$ and $\lceil k\rceil \triangleq \bmod$ $(k-1, N)+1$. The constant vorticity or divergence case corresponds to $M_{C}=0$ or $M_{D}=0$, respectively. The case when the vorticity or divergence is assumed to be identically zero will be denoted by $M_{C}=-1$ or $M_{D}=$ -1 , respectively.

A simple special case occurs for $M_{C}=M_{D}=1$; then,

$$
\begin{aligned}
& \zeta_{i, j}=c_{0.0}+c_{1.0} i+c_{0.1} j \\
& \delta_{i, j}=d_{0,0}+d_{1,0} i+d_{0.1} j
\end{aligned}
$$

so that (48) can be written as

$$
\begin{aligned}
\bar{W}= & F \bar{X}+c_{0,0} \bar{R}^{c}+c_{1,0} \bar{R}_{x}^{c}+c_{0,1} \bar{R}_{y}^{c}+d_{0,0} \bar{R}^{d} \\
& +d_{1,0} \bar{R}_{x}^{d}+c_{0,1} \bar{R}_{y}^{d}
\end{aligned}
$$

where $\bar{R}^{c}, \bar{R}^{d}, \bar{R}_{x}^{c}, \bar{R}_{y}^{c}, \bar{R}_{x}^{d}$, and $\bar{R}_{y}^{d}$ are $2 N^{2}$ vectors with elements $\bar{R}_{k}^{c}, \bar{R}_{k}^{d}, \bar{R}_{x k}^{c}, \bar{R}_{y k}^{c}, \bar{R}_{x k}^{d}$, and $\bar{R}_{y k}^{d}$ defined as

$$
\begin{aligned}
\bar{R}_{k}^{c} & =\sum_{i=1}^{N} \sum_{j=1}^{N} r_{k, j+(i-1) N}^{c} \\
\bar{R}_{k}^{d} & =\sum_{i=1}^{N} \sum_{j=1}^{N} r_{k, j+(i-1) N}^{d} \\
\bar{R}_{x_{k}}^{c} & =\sum_{i=1}^{N} i \sum_{j=1}^{N} r_{k, j+(i-1) N}^{c} \\
\bar{R}_{y k}^{c} & =\sum_{j=1}^{N} j \sum_{i=1}^{N} r_{k, j+(i-1) N}^{c} \\
\bar{R}_{x_{k}}^{d} & =\sum_{i=1}^{N} i \sum_{j=1}^{N} r_{k, j+(i-1) N}^{d} \\
\bar{R}_{y_{k}}^{d} & =\sum_{j=1}^{N} j \sum_{i=1}^{N} r_{k, j+(i-1) N}^{d}
\end{aligned}
$$

where $r_{k, j}^{c}$ and $r_{k, j}^{d}$ are the elements of $R^{c}$ and $R^{d}$, respectively.

To express (64) in a simple form, we define a new $4 N$ +4 parameter vector $\bar{X}_{r}$ by augmenting $\bar{X}$ with $c_{0,0}, c_{1.0}$, $c_{0.1}, d_{0,0}, d_{1,0}$, and $d_{0,1}$; i.e.

$$
\bar{X}_{r}=\left[\begin{array}{c}
\bar{X} \\
\overline{c_{0.0}} \\
c_{1.0} \\
c_{0,1} \\
d_{0,0} \\
d_{1.0} \\
d_{0.1}
\end{array}\right]
$$


and let the $2 N^{2} \times(4 N+4)$ matrix $F_{r}$ be the matrix created by column-augmenting the matrix $F$ with $\bar{R}^{c}, \bar{R}_{x}^{c}$, etc.; i.e.,

$$
F_{r}=\left[F\left|\bar{R}^{c}\right| \bar{R}_{x}^{c}\left|\bar{R}_{y}^{c}\right| \bar{R}^{d}\left|\bar{R}_{x}^{d}\right| \bar{R}_{y}^{d}\right] .
$$

Equation (64) can then be written as

$$
\bar{W}=F_{r} \bar{X}_{r}
$$

It can be shown that the columns of $F$ are linearly independent [16]; hence $F$ is full rank. It follows from the definitions of $R^{c}$ and $R^{d}$ that the columns of $R^{c}$ and $R^{d}$ are linearly independent. We note that $\bar{R}^{c}, \bar{R}^{d}, \bar{R}_{x}^{c}, \bar{R}_{y}^{c}$, $\bar{R}_{x}^{d}$, and $\bar{R}_{y}^{d}$ are linearly independent of each other (for $N$ $>3$ ) and, further, that these vectors are independent of the columns of $F$. It follows that the columns of $F_{r}$ are linearly independent so that $F_{r}$ is full rank; hence there is a unique relationship between a given $\bar{W}$ and the parameters $\bar{X}_{r}$. Given $\bar{W}$, a least-squares estimate of $\bar{X}_{r}$ is

$$
\bar{X}_{r}=F_{r}^{\dagger} \bar{W}
$$

where $F_{r}^{\dagger}$ is the generalized inverse of $F_{r}$. Since the system of equations is overdetermined, $F_{r}^{\dagger}=\left(F_{r}^{T} F_{r}\right)^{-1} F_{r}^{T}$.

The extension of this approach of augmenting the parameters of the vorticity and divergence field models to the boundary conditions for higher-order polynomial orders is straightforward [16].

\section{Parameterizing the Boundary Conditions}

For a given choice of $M_{C}$ and $M_{D}$, the final wind field model has the form of (73); the wind field is a simple linear function of the boundary conditions for $p$ and the parameters of the vorticity and divergence fields. This model is referred to as the normal boundary (NB) wind field model. We now consider a model option which reduces the number of unknowns in the model parameter vector.

Early in the testing of this wind field model it became apparent that, since the geostrophic pressure field sampled at the scatterometer resolution tends to be relatively smooth, the number of unknown boundary values can be reduced, at a cost of accuracy, by parameterizing the geostrophic pressure field around the region's boundary. While not a required part of our wind field model, minimizing the number of unknown parameters in the model significantly reduces the CPU time required to determine the optimum model parameters when our model is applied to wind field estimation from wind scatterometer measurements.

Since the boundary is closed, the pressure field along the boundary will be periodic. We can parameterize the pressure $p$ as a one-dimensional function along the boundary of the region $\mathcal{L}$. We write the pressure field around the boundary as $p(l)$, where $l$ is related to the discretization grid indexes $i$ and $j$ clockwise around the boundary, according to

$$
l= \begin{cases}j, & i=0,0 \leq j \leq N+1, \\ i+N+1, & j=N+1,0<i \leq N+1, \\ 2 N+2-j, & i=N+1,0 \leq j \leq N+1, \\ 4 N+4-i, & j=0,0 \leq i<N+1 .\end{cases}
$$

This formulation provides a one-to-one mapping from $l$ to the region's boundary. Observe that $l$ runs from 0 to $4 N$ +4 .

Since $p(l)$ is relatively "smooth" and must be periodic, a low-order Fourier series representation is appropriate for it; i.e.,

$$
\begin{aligned}
p(l)= & s_{0}+\sum_{k=1}^{M_{l} / 2}\left[s_{k}^{c} \cos \frac{k l \pi}{2(N+1)}\right. \\
& \left.+s_{k}^{s} \sin \frac{k l \pi}{2(N+1)}\right]
\end{aligned}
$$

where $M_{1}$ is the order of the pressure boundary condition model. We have examined other parameterizations [16]. We have already noted that an arbitrary constant can be added to the pressure field without affecting the model formulation so that we can ignore the $s_{0}$ term. This requires that we modify the definition of $F_{1}$ slightly to incorporate the boundary value $p_{0,1}$. Let $F_{1}^{\prime}$ be the $2 N^{2} \times$ $N$ rectangular matrix defined as

$$
F_{1}^{\prime}=\left[\begin{array}{llll}
{ }_{1} A_{1} & { }_{1} A_{2} & \cdots & { }_{1} A_{N} \\
{ }_{4} A_{1} & { }_{4} A_{2} & \cdots & { }_{4} A_{N}
\end{array}\right]=\left[\begin{array}{c}
{ }_{1} A_{1} \\
{ }_{4} A_{1}
\end{array} \mid F_{1}\right] .
$$

Let the $M_{l}$ element vector $\bar{Y}$ be defined as

$$
\bar{Y} \triangleq\left[\begin{array}{c}
s_{1}^{c} \\
s_{1}^{s} \\
s_{2}^{c} \\
s_{2}^{s} \\
\vdots \\
s_{M_{1} / 2}^{c} \\
s_{M_{i} / 2}^{s}
\end{array}\right] .
$$

Equations (40) and (41) can be then be written as

$$
\bar{W}=\mathfrak{F} \bar{Y}+R^{c} C+R^{d} D
$$

where $\mathcal{F}$ is a $2 N^{2} \times M_{l}$ rectangular matrix created from the $F_{j}$ matrices and $F_{1}^{\prime}$. Let $f_{i, j}$ be the $(i, j)$ th element of 
$\mathcal{F}$ and $\left(F_{k}\right)_{i, j}$ be the $(i, j)$ th element of the $F_{k}$ matrix; then

$$
f_{i, j}=\left\{\begin{aligned}
\sum_{k=1}^{N} & \left(F_{1}^{\prime}\right)_{i, k} \cos j k /(2 N+2) \pi \\
& +\sum_{k=1}^{N}\left(F_{2}\right)_{i, k} \cos j(2-k) /(2 N+2) \pi \\
& +\sum_{k=1}^{N}\left(F_{3}\right)_{i, k} \cos j(1+k) /(2 N+2) \pi \\
& +\sum_{k=1}^{N}\left(F_{4}\right)_{i, k} \cos j(3-k) /(2 N+2) \pi \\
& \text { for } j \text { odd } \\
\sum_{k=1}^{N} & \left.F_{1}^{\prime}\right)_{i, k} \sin j k /(2 N+2) \pi \\
& +\sum_{k=1}^{N}\left(F_{2}\right)_{i, k} \sin j(2-k) /(2 N+2) \pi \\
& +\sum_{k=1}^{N}\left(F_{3}\right)_{i, k} \sin j(1+k) /(2 N+2) \pi \\
& +\sum_{k=1}^{N}\left(F_{4}\right)_{i, k} \sin j(3-k) /(2 N+2) \pi, \\
& \text { for } j \text { even. }
\end{aligned}\right.
$$

The final parameterized boundary condition (PBC) wind field model is created by augmenting $\bar{Y}$ with the parameters of the vorticity and divergence field model, as was previously done for the NB model.

\section{Vi. Evaluating the Wind Field Model}

Both the NB and PBC wind field model options have the general form

$$
\bar{W}=F \bar{X}
$$

where $\bar{W}$ contains the components of the sampled wind field over the region $\mathscr{L}, F$ is a known constant matrix, and $\bar{X}$ is the model parameter vector. We now consider how well these models can represent realistic wind fields for different orders of the vorticity and divergence field models and field size $N$.

To evaluate the modeling error: (i) a least-squares fit of the model parameters to a real wind field was obtained; (ii) the resulting "'model"' wind field was computed from the model parameters; and (iii) the rms difference between the true field and model field was computed.

The sampled "true" wind field over $\mathcal{L}$ is denoted by $\bar{W}_{t}$. The least-squares fit $\bar{X}$ of the model parameters to $\bar{W}_{t}$ is

$$
\bar{X}=F^{\dagger} \bar{W}_{t}
$$

where $F^{\dagger}=\left(F^{T} F\right)^{-1} F^{T}$ is the pseudoinverse of $F$. The wind field computed from the model parameter vector,

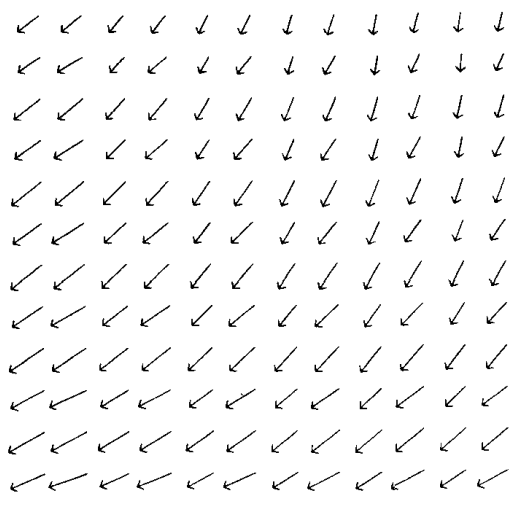

Fig. 3. An example of a wind ficld uniformly sampled, with $h=25 \mathrm{~km}$ over a $300 \times 300 \mathrm{~km}$ region. A vector length equal to the sample spacing corresponds to $15 \mathrm{~m} / \mathrm{s}$.

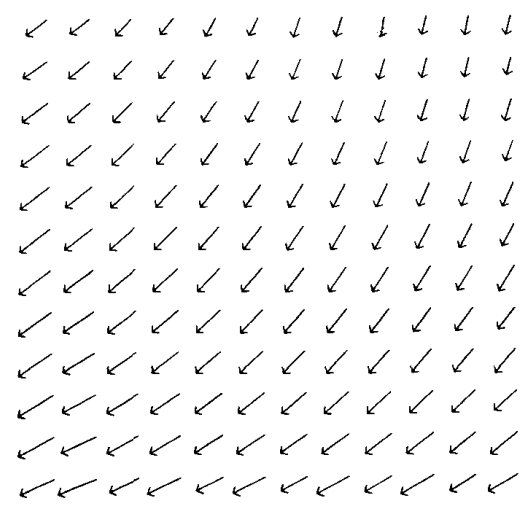

Fig. 4. The "model" wind field resulting from fitting the NB model with $M_{C}=M_{D}=4$ and $N=12$ to the wind field shown in Fig. 3. Plotting conventions and scale are the same as in Fig. 3. The model fit error is given in Table II (see text).

denoted $\bar{W}$, is

$$
\bar{W}=F \bar{X} .
$$

The vector error between $\bar{W}_{t}$ and $\bar{W}$ is then

$$
\bar{W}_{t}-\bar{W}=\left(I-F F^{\dagger}\right) \bar{W}_{t}=\left(I-F\left(F^{T} F\right)^{-1} F^{T}\right) \bar{W}_{t} .
$$

To illustrate the model performance, consider Figs. 3 and 4. A simulated (described below) mesoscale wind field, sampled at $25 \mathrm{~km}$ with $N=12$, is shown in Fig. 3 . A vector length equal to the distance between samples corresponds to a wind speed of $15 \mathrm{~m} / \mathrm{s}$. The model parameter vector $\bar{X}$ was computed using (82). The model wind field $\bar{W}$ was then computed using (83) and is plotted in Fig. 4. For this example, the NB model was used with $M_{C}=M_{D}=2$. The rms differences between $\bar{W}_{t}$ and $\bar{W}$ are tabulated in Table II. In this and succeeding tables, the rms vector error is defined as the square root of the mean-squared magnitude of the vector difference between the true field and estimated field. The value shown is nor- 


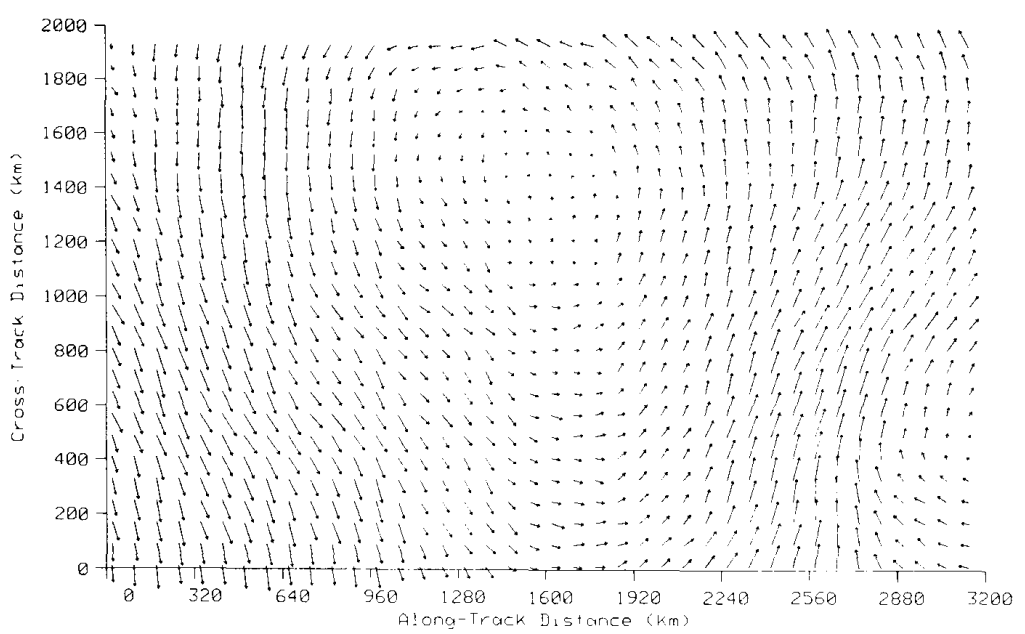

Fig. 5. An example of the mesoscale wind fields used in evaluating the model accuracy. The field was uniformly sampled with $h=80 \mathrm{~km}$. A vector length equal to the sample spacing corresponds to $15 \mathrm{~m} / \mathrm{s}$.

TABLE II

RMS Difference Between the True Wind Field in Fig. 3 and the, FIELD IN FIG. 4

\begin{tabular}{c|c|c}
\hline $\begin{array}{c}\text { Normalized } \\
\text { Vector }\end{array}$ & Direction (deg) & $\begin{array}{c}\text { Normalized } \\
\text { Speed }\end{array}$ \\
\hline 0.09 & 4.29 & 0.06 \\
\hline
\end{tabular}

malized by the rms vector magnitude of the true wind field. Similarly, the rms wind speed error has been normalized by the rms wind speed of the true wind field. Note the close agreement between the true and model wind fields. We note that for small values of $M_{C}$ and $M_{D}$, the model field will be a "smoothed" version of the true field. The amount of smoothing can be controlled by selection of the values of $M_{C}$ and $M_{D}$.

To evaluate our model formulation we have used simulated mesoscale wind fields, since little conventional mesoscale wind field data over the ocean are available. The test wind fields are the standard fields used to evaluate NSCAT performance [24]. They were generated by state-of-the-art numerical weather prediction models at $1.875^{\circ}$ resolution. The surface wind fields were interpolated to $10 \mathrm{~km}$ and nondivergent small-scale variability with a $a k^{-2}$ spectrum. For a given $2000 \times 2000 \mathrm{~km}$ region, the value of $a$ was selected to be consistent with the spectrum within the region (see [8]). The wind fields were selected to span a wide range of meteorological conditions, including fronts [24]. An example of a portion of one of the test fields is shown in Fig. 5. Regions of high vorticity and nonzero divergence are readily observable. The sampling interval is $80 \mathrm{~km}$, with a vector length corresponding to the sampling distance equivalent to $15 \mathrm{~m} / \mathrm{s}$.

To evaluate the modeling error for a wind field model of size $N$, each wind field was segmented into $N \times N$ regions. For each region segment the model parameters were computed using the approach described above, and the model wind field was computed from the model parameters. The rms of the error between the true and model fields was computed over all possible regions within the original true wind field. The results for various model options are described below.

As a general rule for fixed $N$, as $M_{C}$ and $M_{D}$ are increased the modeling error is reduced. For given values of $M_{C}$ and $M_{D}$, as $N$ is increased the modeling error increases. Since the number of parameters is a function of $M_{C}, M_{D}$, and $N$, there is room for tradeoff between the number of model parameters and accuracy of the wind field model. We will be primarily interested in values of $N$ such as 8 or 12 , which evenly divide the number of scatterometer measurements across the swath.

\section{A. NB Model Error}

Let us first consider the performance of the NB model. For the NB model the number of unknowns $N_{u}$ in each $N$ $\times N$ region segment is related to $N, M_{C}$, and $M_{D}$ by the formula,

$$
N_{u}=4 N-2+g\left(M_{C}\right)+g\left(M_{D}\right)
$$

where

$$
g(M)= \begin{cases}0, & M<0 \\ (M+1)(M+2) / 2, & M \geq 0 .\end{cases}
$$

$M_{C}=-1$ is used to denote the case when the vorticity field is identically zero. Similarly, $M_{D}$ denotes the case when the divergence is identically zero, which would result in a strictly geostrophic model.

Table III shows rms modeling error versus $N$ for polynomial vorticity and divergence models, with $M_{C}=M_{D}$ $=2$. With the exception of a peak at $N=10$, the modeling error increases as $N$ increases. Table IV illustrates the effects of varying $M_{C}$ and $M_{D}$ for $N=8$. Table $\mathrm{V}$ is similar to Table IV, but for $N=12$. As $M_{C}$ and $M_{D}$ increase, the modeling error is reduced. To minimize the number of unknowns in the model, we desire to keep $M_{C}$ and $M_{D}$ small. While other values of $M_{C}$ and $M_{D}$ can be 
TABLE III

WIND-FIELD-Model ERror FOR THE NB MODEL, WITH $M_{C}=M_{D}=2$, AS a Function of $N$ for SimUlated Mesoscale Wind Fields

\begin{tabular}{c|c|c|c|c}
\hline \hline$N$ & $\begin{array}{c}\text { Number of Unknowns } \\
\text { in } N \times N \text { Region }\end{array}$ & \multicolumn{3}{|c}{ Normalized RMS Error } \\
Vector & Direction (deg) & Speed \\
\hline 4 & 20 & 0.024 & 1.06 & 0.011 \\
6 & 28 & 0.080 & 3.11 & 0.046 \\
8 & 36 & 0.084 & 3.69 & 0.050 \\
10 & 44 & 0.104 & 4.92 & 0.065 \\
12 & 54 & 0.083 & 4.27 & 0.052 \\
14 & 66 & 0.082 & 4.48 & 0.054 \\
16 & 74 & 0.084 & 5.04 & 0.057 \\
\hline
\end{tabular}

TABLE IV

NB-Model ERROR FOR $N=8$, verSUS $M_{C}$ AND $M_{D}$

\begin{tabular}{|c|c|c|c|c|c|}
\hline \multicolumn{2}{|c|}{ Field Model } & \multirow{2}{*}{$\begin{array}{c}\text { Unknowns } \\
N_{u}\end{array}$} & \multicolumn{3}{|c|}{ Normalized RMS Error } \\
\hline$M_{C}$ & $M_{D}$ & & Vector & Direction (deg) & Speed \\
\hline-1 & -1 & 30 & $0.1 \overline{9} \overline{1}$ & 8.31 & 0.115 \\
\hline-1 & 0 & 31 & 0.171 & 7.22 & 0.099 \\
\hline-1 & 1 & 33 & 0.165 & 6.97 & 0.095 \\
\hline-1 & 2 & 36 & 0.163 & 6.95 & 0.093 \\
\hline-1 & 3 & 40 & 0.158 & 6.74 & 0.091 \\
\hline-1 & 4 & 45 & 0.144 & 6.17 & 0.084 \\
\hline 0 & -1 & 31 & 0.133 & 6.01 & 0.084 \\
\hline 0 & 0 & 32 & 0.102 & 4.65 & 0.063 \\
\hline 0 & 1 & 34 & 0.096 & 4.36 & 0.059 \\
\hline 0 & 2 & 37 & 0.095 & 4.30 & 0.058 \\
\hline 0 & 3 & 41 & 0.095 & 4.27 & 0.058 \\
\hline 0 & 4 & 46 & 0.094 & 4.21 & 0.057 \\
\hline 1 & -1 & 33 & 0.129 & 5.79 & 0.080 \\
\hline 1 & 0 & 34 & 0.097 & 4.35 & 0.058 \\
\hline 1 & 1 & 36 & 0.090 & 4.01 & 0.054 \\
\hline 1 & 2 & 39 & 0.090 & 3.96 & 0.053 \\
\hline 1 & 3 & 43 & 0.089 & 3.92 & 0.053 \\
\hline 1 & 4 & 48 & 0.089 & 3.91 & 0.053 \\
\hline 2 & -1 & 36 & 0.125 & 5.62 & 0.077 \\
\hline 2 & 0 & 37 & 0.092 & 4.09 & 0.055 \\
\hline 2 & 1 & 39 & 0.085 & 3.72 & 0.050 \\
\hline 2 & 2 & 42 & 0.084 & 3.69 & 0.050 \\
\hline 2 & 3 & 46 & 0.084 & 3.68 & 0.050 \\
\hline 2 & 4 & 51 & 0.084 & 3.67 & 0.050 \\
\hline 3 & -1 & 40 & 0.125 & 5.60 & 0.077 \\
\hline 3 & 0 & 41 & 0.091 & 4.06 & 0.055 \\
\hline 3 & 1 & 43 & 0.084 & 3.70 & 0.050 \\
\hline 3 & 2 & 46 & 0.084 & 3.66 & 0.049 \\
\hline 3 & 3 & 50 & 0.084 & 3.65 & 0.049 \\
\hline 3 & 4 & 55 & 0.084 & 3.64 & 0.049 \\
\hline 4 & -1 & 45 & 0.118 & 5.30 & 0.073 \\
\hline 4 & 0 & 46 & 0.082 & 3.68 & 0.049 \\
\hline 4 & 1 & 48 & 0.074 & 3.25 & 0.044 \\
\hline 4 & 2 & 51 & 0.074 & 3.20 & 0.043 \\
\hline 4 & 3 & 55 & 0.074 & 3.20 & 0.043 \\
\hline 4 & 4 & 60 & 0.073 & 3.20 & 0.043 \\
\hline
\end{tabular}

chosen, our desired accuracy requirements (see Section II) will be met for $N=8$, with $M_{C}=M_{D}=0$; i.e., for a constant vorticity and divergence model over the region $\mathcal{L}$, whereas for $N=12, M_{C}=M_{D}=1$ will meet the desired requirements.

\section{B. PBC Model Error}

The PBC model has the advantage of using a smaller number of unknowns than the NB model, but at the expense of a somewhat higher modeling error. For the PBC model the number of unknowns $N_{u}$ in each $N \times N$ region is related to $M_{l}, M_{C}$, and $M_{D}$ by the formula,

$$
N_{u}=M_{l}+g\left(M_{C}\right)+g\left(M_{D}\right) \text {. }
$$

Next we contrast the performance of the previous NB model results with those obtained for the PBC model. Table VI illustrates the effect of varying $M_{l}$ for $N=8$ and
TABLE $\mathrm{V}$

\begin{tabular}{|c|c|c|c|c|c|}
\hline \multicolumn{2}{|c|}{ Field Model } & \multirow{2}{*}{$\begin{array}{c}\text { Unknowns } \\
N_{u}\end{array}$} & \multicolumn{3}{|c|}{ Normalized RMS Error } \\
\hline$M_{C}$ & $M_{D}$ & & Vector & Direction (deg) & Speed \\
\hline-1 & -1 & 46 & 0.234 & 11.21 & 0.148 \\
\hline-1 & 0 & 47 & 0.208 & 9.76 & 0.127 \\
\hline-1 & 1 & 49 & 0.199 & 9.23 & 0.120 \\
\hline-1 & 2 & 52 & 0.197 & 9.12 & 0.118 \\
\hline-1 & 3 & 56 & 0.196 & 9.07 & 0.117 \\
\hline-1 & 4 & 61 & 0.193 & 9.05 & 0.116 \\
\hline 0 & -1 & 47 & 0.163 & 8.03 & 0.108 \\
\hline 0 & 0 & 48 & 0.123 & 6.26 & 0.082 \\
\hline 0 & 1 & 50 & 0.109 & 5.63 & 0.073 \\
\hline 0 & 2 & 53 & 0.106 & 5.46 & 0.070 \\
\hline 0 & 3 & 57 & 0.105 & 5.44 & 0.069 \\
\hline 0 & 4 & 62 & 0.107 & 5.55 & 0.071 \\
\hline 1 & -1 & 49 & 0.152 & 7.56 & 0.098 \\
\hline 1 & 0 & 50 & 0.108 & 5.60 & 0.070 \\
\hline 1 & 1 & 52 & 0.092 & 4.77 & 0.059 \\
\hline 1 & 2 & 55 & 0.088 & 4.56 & 0.056 \\
\hline 1 & 3 & 59 & 0.087 & 4.52 & 0.056 \\
\hline 1 & 4 & 64 & 0.087 & 4.53 & 0.056 \\
\hline 2 & -1 & 52 & 0.149 & 7.44 & 0.096 \\
\hline 2 & 0 & 53 & 0.104 & 5.43 & 0.067 \\
\hline 2 & 1 & 55 & 0.087 & 4.52 & 0.055 \\
\hline 2 & 2 & 58 & 0.083 & 4.27 & 0.052 \\
\hline 2 & 3 & 62 & 0.083 & 4.23 & 0.051 \\
\hline 2 & 4 & 67 & 0.083 & 4.24 & 0.052 \\
\hline 3 & -1 & 56 & 0.149 & 7.42 & 0.095 \\
\hline 3 & 0 & 57 & 0.103 & 5.40 & 0.066 \\
\hline 3 & 1 & 59 & 0.086 & 4.49 & 0.055 \\
\hline 3 & 2 & 62 & 0.083 & 4.23 & 0.051 \\
\hline 3 & 3 & 66 & 0.082 & 4.19 & 0.051 \\
\hline 3 & 4 & 71 & 0.086 & 4.54 & 0.054 \\
\hline 4 & -1 & 61 & 0.147 & 7.34 & 0.094 \\
\hline 4 & 0 & 62 & 0.101 & 5.26 & 0.065 \\
\hline 4 & 1 & 64 & 0.085 & 4.46 & 0.054 \\
\hline 4 & 2 & 67 & 0.082 & 4.30 & 0.052 \\
\hline 4 & 3 & 71 & 0.080 & 4.13 & 0.050 \\
\hline 4 & 4 & 76 & 0.080 & 4.10 & 0.050 \\
\hline
\end{tabular}

TABLE VI

PBC-Model ERror for $N=8$ AND $M_{C}=M_{D}=2$, versus $M_{\ell}$

\begin{tabular}{c|c|c|c|c}
\hline \hline$M_{l}$ & Unknowns & \multicolumn{3}{|c}{ Normalized RMS Error } \\
& $N_{u}$ & Vector & Direction (deg) & Speed \\
\hline 2 & 14 & 0.237 & 10.98 & 0.153 \\
4 & 16 & 0.160 & 7.48 & 0.111 \\
6 & 18 & 0.116 & $\mathbf{5 . 1 1}$ & 0.076 \\
8 & 20 & 0.106 & 4.80 & 0.068 \\
10 & 22 & 0.097 & 4.40 & 0.059 \\
12 & 24 & 0.096 & 4.32 & 0.058 \\
\hline
\end{tabular}

TABLE VII

PBC-Model ERror, With $M_{l}=8$ AND $M_{C}=M_{D}=2$, AS A Function OF $N$ for Simulated Mesoscale Wind Fields

\begin{tabular}{c|c|c|c|c}
\hline \hline$N$ & $\begin{array}{c}\text { Number of Unknowns } \\
\text { in } N \times N \text { Region }\end{array}$ & \multicolumn{3}{|c}{ Normalized RMS Error } \\
& Vector & Direction (deg) & Speed \\
\hline $\mathbf{4}$ & 20 & 0.057 & 2.14 & 0.032 \\
6 & 20 & 0.099 & 4.12 & 0.060 \\
8 & 20 & 0.106 & 4.80 & 0.068 \\
10 & 20 & 0.107 & 5.02 & 0.072 \\
$\mathbf{1 2}$ & 20 & 0.110 & 5.64 & 0.075 \\
$\mathbf{1 4}$ & 20 & 0.112 & 5.91 & 0.078 \\
$\mathbf{1 6}$ & 20 & 0.116 & 6.56 & 0.082 \\
\hline
\end{tabular}

$M_{C}=M_{D}=2$. Table VII presents the rms errors for $M_{l}$ $=8$ and $M_{C}=M_{D}=2$ versus $N$. Table VII should be compared to Table III; note that the errors are only slightly larger for the PBC case as compared to the NB case. Table VIII shows the effects of varying $M_{C}$ and $M_{D}$ for $N=$ 8 and $M_{l}=8$, whereas Table IX presents similar results for $N=12$. Table VIII should be compared with Table 


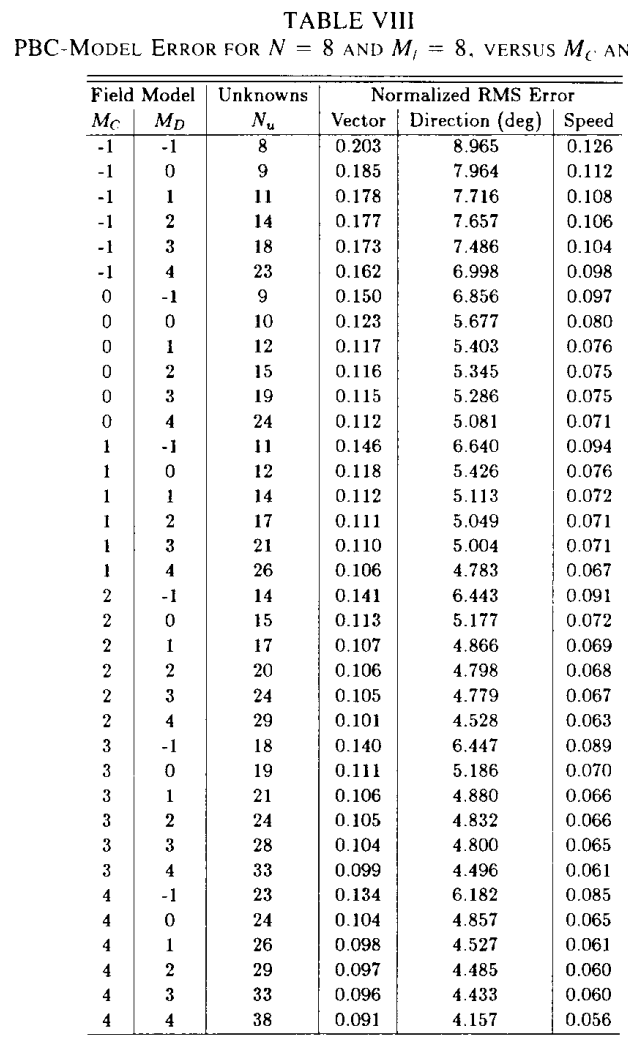

V, whereas Table IX should be compared with Table VI. Observe that for $N=8$, setting $M_{l}=8$ and $M_{C}=M_{D}=$ 1 permits us to meet our desired model accuracy requirements. For $N=12$ and $M_{l}=8$ and $M_{C}, M_{D}=2$, the desired accuracy requirements are met. Greater accuracy is achieved for larger $M_{l}$ and/or larger $M_{C}$ and $M_{D}$.

\section{CONCLUSIONS}

In this paper we have developed a model for near-surface mesoscale wind fields which is suitable for use in the model-based estimation of wind fields from wind scatterometer measurements. The model can accurately describe near-surface mesoscale wind fields, is based only on scatterometer data (i.e., no other instrument or in situ data is required), is computationally tractable, and lends itself to a model parameter estimation formulation. The modeling error has been evaluated by means of simulation. We have found that a parameterized boundary condition model with $N=8, M_{l}=8$, and $M_{C}=M_{D}=1$ or $N=12, M_{l}=8$, and $M_{C}=M_{D}=2$ provides the desired model accuracy while minimizing the number of unknowns. While we have developed our wind field model expressly for wind field estimation from scatterometer measurements, it is our hope that these results may prove helpful in other research areas.

A companion paper (Part II [26]) describes the application of our model to wind field estimation from scatterometer measurements.

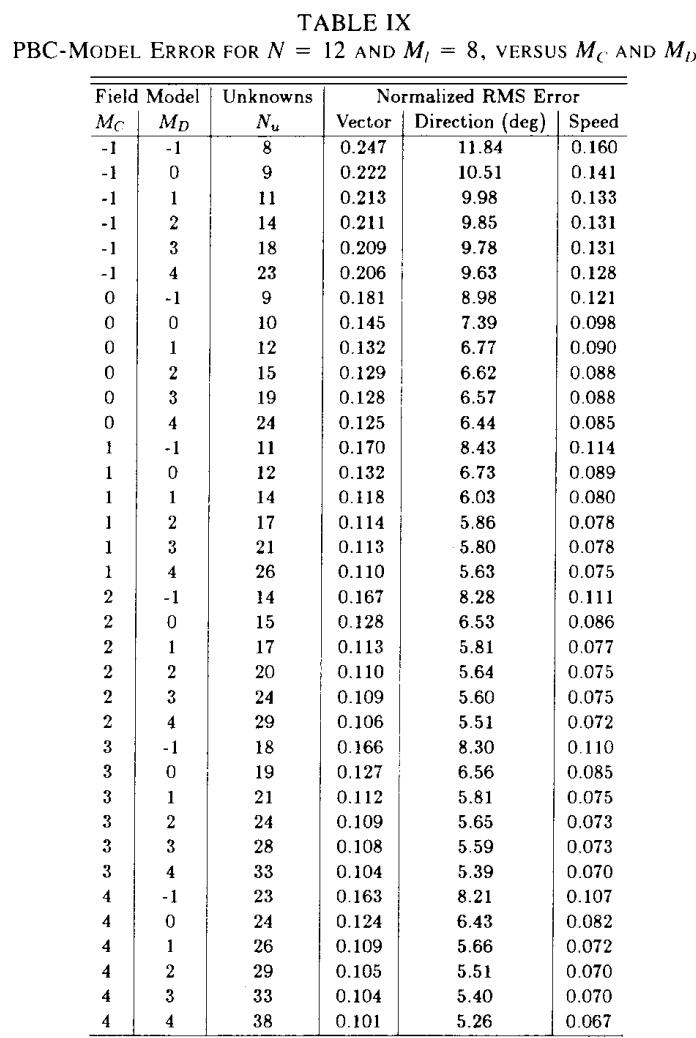

\section{ACKNOWLEDGMENT}

The authors acknowledge the assistance of Dr. M. Freilich of NASA's Jet Propulsion Laboratory, who supplied the mesoscale wind fields used in evaluating the wind field model.

\section{REFERENCES}

[1] S. J. Bijlsma, L. M. Hafkenscheid, and P. Lynch, "Computation of the streamfunction and velocity potential and reconstruction of the wind field," Monthly Weather Rev., vol. 114, pp. 1547-1551, 1986.

[2] E. Bracalente, D. Boggs, W. Grantham, and J. Sweet, "The SASS scattering coefficient $\left(\sigma_{0}\right)$ algorithm," IEEE J. Oceanic Eng., vol OE-5, pp. 145-154, Apr. 1980.

[3] J. W. Brewer, "Kronecker products and matrix calculus in system theory,"'IEEE Trans. Circuits Syst., vol. CAS-25, pp. 772-781, Sept 1978.

[4] R. A. Brown et al., "Surface wind analyses for SEASAT," J. Geophys. Res., vol. 87, pp. 3355-3364, Apr. 1982.

[5] C. J. Cats, "Analysis of surface wind and its gradient in a mesoscale wind observation network," Monthly Weather Rev., vol. 108, pp. 1100-1107, Aug. 1980.

[6] M. H. Freilich, "Science opportunities using the NASA scatterometer on N-ROSS," Jet Propulsion Lab., Pasadena, CA, JPL Pub. 87 57, Feb. 1, 1985.

[7] M. H. Freilich, "Satellite scatterometer comparisons with surface measurements: Techniques and SEASAT results," in Proc. Workshop ERS-1 Wind and Wave Calibration, June 2-6, 1986, ESA SP-262, pp. 57-62

[8] M. H. Freilich and D. B. Chelton, "Wavenumber spectra of Pacific winds measured by the Seasat scatterometer," J. Phys. Oceanogr., vol. 16, pp. 741-757, Apr. 1986.

[9] R. N. Hoffman, "SASS wind ambiguity removal by direct minimization," Monthly Weather Rev., vol. 110, pp. 434-445, May 1982.

[10] R. N. Hoffman, "SASS wind ambiguity removal by direct minimi- 
zation. Part Il: Use of smoothness and dynamical constraints,' Monthly Weather Rev., vol. 112, pp. 1829-1852, Sept. 1982.

[11] A. K. Jain and J. R. Jain, "Partial differential equations and finite difference methods in image processing-Part II: Image restoration," IEEE Trans. Automat. Contr., vol. AC-23, pp. 817-834, Oct. 1978

[12] W. L. Jones et al., "The SEASAT-A satellite scatterometer: The geophysical evaluation of remotely sensed wind vectors over the ocean," J. Geophys. Res., vol. 87, pp. 3297-3317, Apr. 1982.

[13] F. Li, P. Callahan, M. Freilich, D. Long, and C. Winn, "NASA scatterometer for NROSS-A system for global oceanic wind measurement," in Proc. Int. Geosci. Remote Sensing Symp. (Strasborg, France), 1984, pp. 777-780.

[14] L. Ljung, System Identification Theory for the User. Englewood Cliffs, NJ: Prentice-Hall, 1987.

[15] D. G. Long and J. M. Mendel, "Model-based estimation of wind fields over the ocean from wind scatterometer measurements," in Proc. Int. Geosci. Remote Sensing Symp. (Edinburgh, Scotland), 1988, pp. 553-556.

[16] D. G. Long, "Model-based estimation of wind fields over the ocean from wind scatterometer measurements," Ph.D. dissertation, University of Southern California, Los Angeles, 1989.

[17] P. Lynch, "Deducing the wind from vorticity and divergence," Monthly Weather Rev., vol. 116, pp. 86-93, Jan. 1988

[18] J. M. Mendel, Discrete Techniques of Parameter Estimation. New York: Marcel Dekker, 1973.

[19] A. R. Mitchell, Computational Methods in Partial Differential Equations. London: Wiley, 1969.

[20] S. V. Parter, "Remarks on the numerical computation of solutions of $\Delta u=f(P, u)$, , in Numerical Solution of Partial Differential Equations, J. H. Bramble, Ed. New York: Academic, 1966.

[21] J. O'Brien, Scientific Opportunities Using Satellite Wind Stress Mea surements Over the Ocean. Fort Lauderdale, FL: Nova University Press/N.Y.I.T. Press, 1982, NASS Contract Number NAS5-26714.

[22] W. J. Pierson, Jr., W. B. Sylvester, and R. E. Salfi, "Vector wind, horizontal divergence, wind stress, and wind stress curl from SEASAT-SASS at a one degree resolution," in Proc. URSI Comm. F Symp. Workshop (Shoresh, Israel), May 14-23, 1984, NASA Conf. Pub. 2303, pp. 557-565.

[23] J. Pedlosky, Geophysical Fluid Dynamics. New York: SpringerVerlag, 1979.

[24] S. Shaffer, R. S. Dunbar, V. Hsiao, and D. G. Long, "A medianfilter-based ambiguity removal algorithm for NSCAT," IEEE Trans. Geosci. Remote Sensing, to be published.

[25] F. T. Ulaby, R. K. Moore, and A. K. Fung, Microwave Remote Sensing-Active and Passive. Reading, MA: Addison-Wesley, 1981.

[26] D. G. Long and J. M. Mendel, "Model-based estimation of wind fields over the ocean from wind scatterometer measurements, Part II: Model parameter estimation,"'IEEE Trans. Geosci. Remote Sensing, this issue, pp. 361-373.

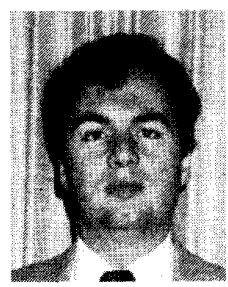

David G. Long (S'80-M'82) received the B.S. and M.S. degrees in electrical engineering from Brigham Young University, Provo, UT, in 1982 and 1983, respectively, and the Ph.D. degree in electrical engineering from the University of Southern California, Los Angeles, in 1989

He has worked for the Jet Propulsion Laboratory, Pasadena, CA, as a Radar Systems Engineer since 1983. He is currently the Project Engineer for the NASA Scatterometer (NSCAT) project and the Experiment Manager for the Eos SCAN-
SCAT. NSCAT is a spaceborne scatterometer designed to measure oceanic winds from space. NSCAT is planned for flight in 1995 aboard the Japanese Advanced Earth Observing System (ADEOS). SCANSCAT is a de velopment scatterometer being studied for flight on NASA's Earth Observing System (Eos) in the late 1990's. He is responsible for the high level design, analysis, and technical management of these projects to insure that the projects meet all mission requirements. His responsibilities include overseeing instrument design and fabrication, algorithm, and coding for the ground processing system, mission operations, and data analysis. His technical responsibilities include system performance analysis, high-level design, development and maintenance of system requirements, and supervision of the Systems Engineering staff. He is also a Group Leader, supervising a staff of system engineers working on a number of JPL flight projects. His research interests include computer graphics, speech and signal processing, estimation theory, radar, and mesoscale atmospheric dynamics.

Dr. Long has received the NASA Award of Achievement several times.

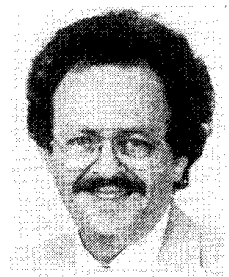

Jerry M. Mendel (S'59-M'61-SM'72-F'78) received the B.S. degree in mechanical engineering and the M.S. and Ph.D. degrees in electrical engineering from the Polytechnic Institute of Brooklyn, Brooklyn, NY, in 1959, 1960, and 1963, respectively.

His experience has included teaching course in electrical engineering at the Polytechnic Institute of Brooklyn from 1960 to 1963 , and has also included various consulting positions. From July 1963 to January 1974 he was with the McDonnell-Douglas Astronautics Company. Currently, he is Professor and Chairman of Electrical Engineering-Systems at the University of Southern California, Los Angeles. He teaches courses in estimation theory and seismic data processing for oil exploration, and was Director of the USC GeoSignal Processing Program (1980-1983). He has published over 230 technical papers and is the author of the monographs: Maximum-Likelihood Deconvolution: A Journey into Model-Based Signal Processing (SpringerVerlag, 1990) and Optimal Seismic Deconvolution: An Estimation-Based Approach (Academic, 1983); the texts: Lessons in Digital Estimation Theory (Prentice-Hall, 1987) and Discrete Techniques of Parameter Estimation: The Equation Error Formulation (Dekker, 1973), and is co-editor (with K. S. Fu) of Adaptive, Learning and Pattern Recognition Systems (Academic, 1970). He is also author of the IEEE Individual Learning Program, Kalman Filtering, and Other Digital Estimation Techniques. He served as Editor of the IEEE Control Systems Society's IEEE TRANSAC TIONS ON AUTOMATIC CONTROL, for which he presently serves as an Associate Editor-at-Large, and is on the Editorial Board of the IEEE PROCEEDINGS.

Dr. Mendel is a member of the Society of Exploration Geophysicists the European Association for Signal Processing, Tau Beta Pi, and Pi Tau Sigma, and is a registered Professional Control Systems Engineer in California. He was President of the IEEE Control Systems Society in 1986. He received the SEG 1976 Outstanding Presentation Award for a paper on the application of Kalman Filtering to deconvolution, the 1983 Best Transactions Paper Award for a paper on maximum-likelihood deconvolution in the IEEE Transactions on Geoscience and Remote Sensing, a Phi Kappa Phi book award for his research monograph on seismic deconvolution, a 1985 Burlington Northern Faculty Achievement Award, and a 1984 IEEE Centennial Medal. 